Proyecciones Journal of Mathematics

Vol. 36, No 4, pp. 601-614, December 2017.

Universidad Católica del Norte

Antofagasta - Chile

\title{
Some results on SD-Prime cordial labeling
}

\author{
A. Lourdusamy \\ St. Xavier's College (Autonomous), India \\ and \\ F. Patrick \\ St. Xavier's College (Autonomous), India \\ Received : February 2017. Accepted : March 2017
}

\begin{abstract}
Given a bijection $f: V(G) \rightarrow\{1,2, \cdots,|V(G)|\}$, we associate 2 integers $S=f(u)+f(v)$ and $D=|f(u)-f(v)|$ with every edge uv in $E(G)$. The labeling $f$ induces an edge labeling $f^{\prime}: E(G) \rightarrow\{0,1\}$ such that for any edge uv in $E(G), f^{\prime}(u v)=1$ if $\operatorname{gcd}(S, D)=1$, and $f^{\prime}(u v)=0$ otherwise. Let $e_{f^{\prime}}(i)$ be the number of edges labeled with $i \in\{0,1\}$. We say $f$ is $S D$-prime cordial labeling if $\left|e_{f^{\prime}}(0)-e_{f^{\prime}}(1)\right| \leq$ 1. Moreover $G$ is $S D$-prime cordial if it admits SD-prime cordial labeling. In this paper, we investigate the SD-prime cordial labeling of some derived graphs.
\end{abstract}

AMS Subject Classification 2010 : $05 C 78$

Keywords : SD-prime labeling, SD-prime cordial labeling, star. 


\section{Introduction}

Let $G=(V(G), E(G))$ be a simple, finite and undirected graph of order $|V(G)|=p$ and size $|E(G)|=q$. All notations not defined in this paper can be found in [2]. A labeling of a graph is a map that carries the graph elements to the set of numbers, usually to the set of non-negative or positive integers. If the domain is the set of vertices the labeling is called vertex labeling. If the domain is the set of edges, then we called about edge labeling. If the labels are assigned to both vertices and edges then the labeling is called total labeling. For all detailed survey of graph labeling we to refer Gallian [1]. In $[4,5]$, G.C. Lau and W.C. Shiu have introduced the concepts SD-prime labeling. In [3], G. C. Lau et.al. have introduced SD-prime cordial labeling and they proved behaviour of several graphs like path, complete bipartite graph, star, double star, wheel, fan, double fan and ladder are SD-prime cordial labeling. In this paper, we investigate the SD-prime cordial labeling behavior of $S^{\prime}\left(K_{1, n}\right), D_{2}\left(K_{1, n}\right)$, $S\left(K_{1, n}\right), D S\left(K_{1, n}\right), S^{\prime}\left(B_{n, n}\right), D_{2}\left(B_{n, n}\right), D S\left(B_{n, n}\right), S\left(B_{n, n}\right), K_{1,3} * K_{1, n}$, $C H_{n}, F l_{n}, P_{n}^{2}, T\left(P_{n}\right), T\left(C_{n}\right)$, the graph obtained by duplication of each vertex of path and cycle by an edge, $Q_{n}, A\left(T_{n}\right), T L_{n}, P_{n} \odot K_{1}, C_{n} \odot K_{1}$ and $J_{n}$.

In [4], Lau and Shiu introduced a variant of prime graph labeling which is defined as follows.

Given a bijection $f: V(G) \rightarrow\{1,2, \cdots,|V(G)|\}$, we associate 2 integers $S=f(u)+f(v)$ and $D=|f(u)-f(v)|$ with every edge $u v$ in $E$.

Definition 1.1. [4] A bijection $f: V(G) \rightarrow\{1,2, \cdots,|V(G)|\}$ induces an edge labeling $f^{\prime}: E(G) \rightarrow\{0,1\}$ such that for any edge $u v$ in $G, f^{\prime}(u v)=1$ if $\operatorname{gcd}(S, D)=1$, and $f^{\prime}(u v)=0$ otherwise. We say $f$ is $S D$-prime labeling if $f^{\prime}(u v)=1$ for all $u v \in E(G)$. Moreover, $G$ is SD-prime if it admits SD-prime labeling.

Definition 1.2. [3] A bijection $f: V(G) \rightarrow\{1,2, \cdots,|V(G)|\}$ induces an edge labeling $f^{\prime}: E(G) \rightarrow\{0,1\}$ such that for any edge $u v$ in $G, f^{\prime}(u v)=1$ if $\operatorname{gcd}(S, D)=1$, and $f^{\prime}(u v)=0$ otherwise. The labeling $f$ is called $S D$ prime cordial labeling if $\left|e_{f^{\prime}}(0)-e_{f^{\prime}}(1)\right| \leq 1$. We say that $G$ is $S D$-prime cordial if it admits $S D$-prime cordial labeling. 
Definition 1.3. For every vertex $v \in V(G)$, the open neighbourhood set $N(v)$ is the set of all vertices adjacent to $v$ in $G$.

Definition 1.4. For a graph $G$ the splitting graph $S^{\prime}(G)$ of a graph $G$ is obtained by adding a new vertex $v^{\prime}$ corresponding to each vertex $v$ of $G$ such that $N(v)=N\left(v^{\prime}\right)$.

Definition 1.5. The shadow graph $D_{2}(G)$ of a connected graph $G$ is obtained by taking two copies of $G$, say $G^{\prime}$ and $G^{\prime \prime}$. Join each vertex $u^{\prime}$ in $G^{\prime}$ to the neighbours of corresponding vertex $u^{\prime \prime}$ in $G^{\prime \prime}$.

Definition 1.6. Duplication of a vertex $v_{k}$ by a new edge $e=v_{k}^{\prime} v_{k}^{\prime \prime}$ in a graph $G$ produces a new graph $G^{\prime}$ such that $N\left(v_{k}^{\prime}\right) \cap N\left(v_{k}^{\prime \prime}\right)=v_{k}$.

Definition 1.7. Let $G$ be the a graph with $V=S_{1} \cup S_{2} \cup S_{3} \cup \cdots S_{t} \cup T$ where each $S_{i}$ is a set of vertices having at least two vertices of the same degree and $T=V(G) \backslash \cup_{i=1}^{t} S_{i}$. The degree splitting graph of $G$ denoted by $D S(G)$ is obtained from $G$ by adding vertices $w_{1}, w_{2}, \ldots, w_{t}$ and joining to each vertex of $S_{i}$ for $1 \leq i \leq t$.

Definition 1.8. For a simple connected graph $G$ the square of graph $G$ is denoted by $G^{2}$ and defined as the graph with the same vertex set as of $G$ and two vertices are adjacent in $G^{2}$ if they are at a distance 1 or 2 apart in $G$.

Definition 1.9. The subdivision graph $S(G)$ is obtained from $G$ by subdividing each edge of $G$ with a vertex.

Definition 1.10. The bistar $B_{n, n}$ is the graph obtained by attaching the apex vertices of two copies of $K_{1, n}$ by an edge.

Definition 1.11. $K_{1,3} * K_{1, n}$ is the graph obtained from $K_{1,3}$ by attaching root of a star $K_{1, n}$ at each pendant vertex of $K_{1,3}$.

Definition 1.12. The triangular ladder is a graph obtained from $L_{n}$ by adding the edges $u_{i} v_{i+1}, 1 \leq i \leq n-1$, where $u_{i}$ and $v_{i}, 1 \leq i \leq n$, are the vertices of $L_{n}$ such that $u_{1}, u_{2}, \cdots, u_{n}$ and $v_{1}, v_{2}, \cdots, v_{n}$ are two paths of order $n$ in the graph $L_{n}$.

Definition 1.13. The corona $G_{1} \odot G_{2}$ of two graphs $G_{1}\left(p_{1}, q_{1}\right)$ and $G_{2}\left(p_{2}, q_{2}\right)$ is defined as the graph obtained by taking one copy of $G_{1}$ and $p_{1}$ copies of $G_{2}$ and joining the $i^{\text {th }}$ vertex of $G_{1}$ with an edge to every vertex in the $i^{\text {th }}$ copy of $G_{2}$. 
Definition 1.14. The total graph $T(G)$ of a graph $G$ is the graph whose vertex set is $V(G) \cup E(G)$ and two vertices are adjacent whenever they are either adjacent or incident in $G$.

\section{Main results}

Theorem 2.1. If $G$ is $S D$-prime cordial of size $q$, then $G-e$ is also $S D$ prime cordial

(i) for all $e \in E(G)$ when $q$ is even.

(ii) for some $e \in E(G)$ when $q$ is odd.

Proof. Case (i): when $q$ is even.

Let $G$ be the SD-prime cordial graph of size $q$, where $q$ is an even number. It follows that $e_{f^{\prime}}(0)=e_{f^{\prime}}(1)=\frac{q}{2}$. Let $e$ be any edge in $G$ which is labeled either 0 or 1 . Then in $G-e$, we have either $e_{f^{\prime}}(0)=e_{f^{\prime}}(1)+1$ or $e_{f^{\prime}}(1)=e_{f^{\prime}}(0)+1$ and hence $\left|e_{f^{\prime}}(0)-e_{f^{\prime}}(1)\right| \leq 1$. Thus $G-e$ is SD-prime cordial for all $e \in E(G)$.

Case (ii): when $q$ is odd.

Let $G$ be the SD-prime cordial graph of size $q$, where $q$ is an odd number. It follows that either $e_{f^{\prime}}(0)=e_{f^{\prime}}(1)+1$ or $e_{f^{\prime}}(1)=e_{f^{\prime}}(0)+1$. If $e_{f^{\prime}}(0)=$ $e_{f^{\prime}}(1)+1$ then remove an edge $e$ which is labeled as 0 and if $e_{f^{\prime}}(1)=$ $e_{f^{\prime}}(0)+1$ then remove an edge $e$ which is labeled as 1 from $G$. It follows that $e_{f^{\prime}}(0)=e_{f^{\prime}}(1)$. Thus, $G-e$ is SD-prime cordial for some $e \in E(G)$.

Corollary 2.2. The graph $G+e$ is $S D$-prime cordial if $G$ is $S D$-prime cordial having even size.

Theorem 2.3. The graph $S^{\prime}\left(K_{1, n}\right)$ is $S D$-prime cordial.

Proof. Let $v_{1}, v_{2}, \cdots, v_{n}$ be the pendant vertices and $v$ be the apex vertex of $K_{1, n}$ and $u, u_{1}, u_{2}, \cdots, u_{n}$ are added vertices corresponding to $v, v_{1}, v_{2}, \cdots, v_{n}$ to obtain $S^{\prime}\left(K_{1, n}\right)$. Therefore, $S^{\prime}\left(K_{1, n}\right)$ is of order $2 n+2$ and size $3 n$. Define $f: V\left(S^{\prime}\left(K_{1, n}\right)\right) \rightarrow\{1,2, \cdots, 2 n+2\}$ as follows: 


$$
\begin{aligned}
& f(v)=4 ; \\
& f(u)=1 ; \\
& f\left(v_{i}\right)=\left\{\begin{array}{lll}
2 i+1 & \text { if } i \text { is odd and } & 1 \leq i \leq n \\
2 i+2 & \text { if } i \text { is even and } & 1 \leq i \leq n
\end{array}\right. \\
& f\left(u_{i}\right)=\left\{\begin{array}{lll}
2 & \text { if } i=1 \\
2 i+2 & \text { if } i \text { is odd and } & 2 \leq i \leq n \\
2 i+1 & \text { if } i \text { is even and } & 2 \leq i \leq n
\end{array}\right.
\end{aligned}
$$

In view of the above labeling pattern we get, $e_{f^{\prime}}(1)=\left\lfloor\frac{3 n}{2}\right\rfloor$ and $e_{f^{\prime}}(0)=$ $\left\lceil\frac{3 n}{2}\right\rceil$.

Thus, $\left|e_{f^{\prime}}(0)-e_{f^{\prime}}(1)\right| \leq 1$. Hence, $S^{\prime}\left(K_{1, n}\right)$ is SD-prime cordial.

Theorem 2.4. The graph $D_{2}\left(K_{1, n}\right)$ is $S D$-prime cordial.

Proof. Let $u, u_{1}, u_{2}, \cdots, u_{n}$ and $v, v_{1}, v_{2}, \cdots, v_{n}$ be the vertices of two copies of $K_{1, n}$. Let $V\left(D_{2}\left(K_{1, n}\right)\right)=\{u, v\} \bigcup\left\{u_{i}, v_{i}: 1 \leq i \leq n\right\}$ and $E\left(D_{2}\left(K_{1, n}\right)\right)=\left\{u u_{i}, v v_{i}, u v_{i}, v u_{i}: 1 \leq i \leq n\right\}$. Therefore, $D_{2}\left(K_{1, n}\right)$ is of order $2 n+2$ and size $4 n$. Define $f: V\left(D_{2}\left(K_{1, n}\right)\right) \rightarrow\{1,2, \cdots, 2 n+2\}$ as follows:

$$
\begin{aligned}
& f(u)=1 ; \\
& f(v)=2 ; \\
& f\left(u_{i}\right)= \begin{cases}2 i+1 & \text { if } i \text { is odd and } 1 \leq i \leq n \\
2 i+2 & \text { if } i \text { is even and } 1 \leq i \leq n ;\end{cases} \\
& f\left(v_{i}\right)= \begin{cases}2 i+2 & \text { if } i \text { is odd and } 1 \leq i \leq n \\
2 i+1 & \text { if } i \text { is even and } 1 \leq i \leq n .\end{cases}
\end{aligned}
$$

In view of the above labeling pattern we get, $e_{f^{\prime}}(0)=e_{f^{\prime}}(1)=2 n$. Thus, $\left|e_{f^{\prime}}(0)-e_{f^{\prime}}(1)\right| \leq 1$. Hence, $D_{2}\left(K_{1, n}\right)$ is SD-prime cordial.

Theorem 2.5. The graph $S\left(K_{1, n}\right)$ is $S D$-prime cordial.

Proof. Let $v, v_{1}, v_{2}, \cdots, v_{n}$ be the vertices of $K_{1, n}$. Let $V\left(S\left(K_{1, n}\right)\right)=$ $\{v\} \bigcup\left\{v_{i}, u_{i}: 1 \leq i \leq n\right\}$ and $E\left(S\left(K_{1, n}\right)\right)=\left\{v u_{i}, v_{i} u_{i}: 1 \leq i \leq n\right\}$. Therefore, $S\left(K_{1, n}\right)$ is of order $2 n+1$ and size $2 n$. Define $f: V\left(S\left(K_{1, n}\right)\right) \rightarrow$ $\{1,2, \cdots, 2 n+1\}$ as follows:

$$
\begin{aligned}
& f(v)=1 ; \\
& f\left(u_{i}\right)=2 i+1,1 \leq i \leq n \\
& f\left(v_{i}\right)=2 i, 1 \leq i \leq n
\end{aligned}
$$


In view of the above labeling pattern we get, $e_{f^{\prime}}(0)=e_{f^{\prime}}(1)=n$. Thus, $\left|e_{f^{\prime}}(0)-e_{f^{\prime}}(1)\right| \leq 1$. Hence $S\left(K_{1, n}\right)$ is SD-prime cordial.

Theorem 2.6. The graph $S^{\prime}\left(B_{n, n}\right)$ is $S D$-prime cordial.

Proof. Let $u_{1}, u_{2}, \cdots, u_{n}, v_{1}, v_{2}, \cdots, v_{n}$ be the pendant vertices and $u, v$ be the apex vertices of $B_{n, n}$. Let $u^{\prime}, v^{\prime}, u_{i}^{\prime}, v_{i}^{\prime}$ be added vertices corresponding to $u, v, u_{i}, v_{i}$ to obtain $S^{\prime}\left(B_{n, n}\right)$. Therefore, $S^{\prime}\left(B_{n, n}\right)$ is of order $4 n+4$ and size $6 n+3$. Define $f: V\left(S^{\prime}\left(B_{n, n}\right)\right) \rightarrow\{1,2, \cdots, 4 n+4\}$ as follows:

$$
\begin{aligned}
& f(u)=6 \text {; } \\
& f(v)=2 \text {; } \\
& f\left(u^{\prime}\right)=3 \text {; } \\
& f\left(v^{\prime}\right)=1 \text {; } \\
& f\left(u_{i}\right)= \begin{cases}4 & \text { if } i=1 \\
4 i+4 & \text { if } i \equiv 1(\bmod 3) \text { and } 2 \leq i \leq n \\
4 i+2 & \text { if } i \equiv 0,2(\bmod 3) \text { and } 2 \leq i \leq n\end{cases} \\
& f\left(u_{i}^{\prime}\right)= \begin{cases}8 & \text { if } i=1 \\
4 i+2 & \text { if } i \equiv 1(\bmod 3) \text { and } 2 \leq i \leq n \\
4 i+4 & \text { if } i \equiv 0,2(\bmod 3) \text { and } 2 \leq i \leq n\end{cases} \\
& f\left(v_{i}\right)=5+4(i-1), 1 \leq i \leq n \text {; } \\
& f\left(v_{i}^{\prime}\right)=7+4(i-1), 1 \leq i \leq n \text {. }
\end{aligned}
$$

In view of the above labeling pattern we get, $e_{f^{\prime}}(0)=\left\lfloor\frac{6 n+3}{2}\right\rfloor$ and $e_{f^{\prime}}(1)=\left\lceil\frac{6 n+3}{2}\right\rceil$.

Thus, $\left|e_{f^{\prime}}(0)-e_{f^{\prime}}(1)\right| \leq 1$. Hence, $S^{\prime}\left(B_{n, n}\right)$ is SD-prime cordial.

Theorem 2.7. The graph $D_{2}\left(B_{n, n}\right)$ is $S D$-prime cordial.

Proof. Let $u_{1}, u_{2}, \cdots, u_{n}, v_{1}, v_{2}, \cdots, v_{n}$ be the pendant vertices and $u, v$ be the apex vertices of $B_{n, n}$. Let

$$
\begin{aligned}
& V\left(D_{2}\left(B_{n, n}\right)\right)=\left\{u, v, u^{\prime}, v^{\prime}\right\} \bigcup\left\{u_{i}, v_{i}, u_{i}^{\prime}, v_{i}^{\prime}: 1 \leq i \leq n\right\} \text { and } \mathrm{E}\left(\mathrm{D}_{2}\left(B_{n, n}\right)\right) \\
& =\left\{\mathrm{uv}, \mathrm{u}^{\prime} v, u v^{\prime}, u^{\prime} v^{\prime}\right\} \bigcup\left\{u u_{i}, u u_{i}^{\prime}, u^{\prime} u_{i}^{\prime}, u^{\prime} u_{i}, v v_{i}, v v_{i}^{\prime}, v^{\prime} v_{i}, v^{\prime} v_{i}^{\prime}: 1 \leq i \leq n\right\} .
\end{aligned}
$$

Therefore, $D_{2}\left(B_{n, n}\right)$ is of order $4 n+4$ and size $8 n+4$. Define $f: V\left(D_{2}\left(B_{n, n}\right)\right) \rightarrow\{1,2, \cdots, 4 n+4\}$ as follows: 
Let $p$ be the highest prime number $<4 n+4$.

$$
\begin{aligned}
& f(u)=1 ; \\
& f(v)=p ; \\
& f\left(u^{\prime}\right)=2 ; \\
& f\left(v^{\prime}\right)=4 ; \\
& f\left(u_{i}^{\prime}\right)=4 i+2, \quad 1 \leq i \leq n ; \\
& f\left(v_{i}\right)=4 i+4,1 \leq i \leq n .
\end{aligned}
$$

For the vertices $u_{1}, u_{2}, \cdots, u_{n}, v_{1}^{\prime}, v_{2}^{\prime}, \cdots, v_{n}^{\prime}$ we assign distinct odd numbers except 1 and $p$. In view of the above labeling pattern we get, $e_{f^{\prime}}(0)=$ $e_{f^{\prime}}(1)=4 n+2$.

Thus, $\left|e_{f^{\prime}}(0)-e_{f^{\prime}}(1)\right| \leq 1$. Hence, $D_{2}\left(B_{n, n}\right)$ is SD-prime cordial.

Theorem 2.8. The graph $D S\left(B_{n, n}\right)$ is $S D$-prime cordial.

Proof. Let $u_{1}, u_{2}, \cdots, u_{n}, v_{1}, v_{2}, \cdots, v_{n}$ be the pendant vertices and $u, v$ be the apex vertices of $B_{n, n}$. Let $V\left(D S\left(B_{n, n}\right)\right)=\left\{u, v, w_{1}, w_{2}\right\} \bigcup\left\{u_{i}, v_{i}\right.$ : $1 \leq i \leq n\}$ and $E\left(D S\left(B_{n, n}\right)\right)=\left\{u v, u w_{2}, v w_{2}\right\} \bigcup\left\{u u_{i}, v v_{i}, u_{i} w_{1}, v_{i} w_{1}: 1 \leq\right.$ $i \leq n\}$. Therefore, $D S\left(B_{n, n}\right)$ is of order $2 n+4$ and size $4 n+3$. Define $f: V\left(D S\left(B_{n, n}\right)\right) \rightarrow\{1,2, \cdots, 2 n+4\}$ as follows:

$$
\begin{aligned}
& f(u)=4 ; \\
& f(v)=2 ; \\
& f\left(w_{1}\right)=1 ; \\
& f\left(w_{2}\right)=2 n+3 ; \\
& f\left(u_{i}\right)=6+2(i-1), 1 \leq i \leq n ; \\
& f\left(v_{i}\right)=3+2(i-1), 1 \leq i \leq n .
\end{aligned}
$$

In view of the above labeling pattern we get, $e_{f^{\prime}}(0)=2 n+1$ and $e_{f^{\prime}}(1)=2 n+2$.

Thus, $\left|e_{f^{\prime}}(0)-e_{f^{\prime}}(1)\right| \leq 1$. Hence, $D S\left(B_{n, n}\right)$ is SD-prime cordial.

Theorem 2.9. The graph $S\left(B_{n, n}\right)$ is $S D$-prime cordial.

Proof. Let $u_{1}, u_{2}, \cdots, u_{n}, v_{1}, v_{2}, \cdots, v_{n}$ be the pendant vertices and $u, v$ be the apex vertices of $B_{n, n}$. Let $v_{i}^{\prime}$ and $u_{i}^{\prime}$ be the newly added vertices between $v$ and $v_{i}$ and $u$ and $u_{i}$ respectively. Also, $w$ be the newly added vertex between $u$ and $v$. Therefore, $S\left(B_{n, n}\right)$ is of order $4 n+3$ and size 
$4 n+2$. Define $f: V\left(S\left(B_{n, n}\right)\right) \rightarrow\{1,2, \cdots, 4 n+3\}$ as follows:

$$
\begin{aligned}
& f(u)=1 ; \\
& f(v)=2 ; \\
& f(w)=3 ; \\
& f\left(u_{i}\right)=4 i, 1 \leq i \leq n ; \\
& f\left(u_{i}^{\prime}\right)=4 i+1,1 \leq i \leq n \\
& f\left(v_{i}\right)=4 i+3,1 \leq i \leq n \\
& f\left(v_{i}^{\prime}\right)=4 i+2,1 \leq i \leq n .
\end{aligned}
$$

In view of the above labeling pattern we get, $e_{f^{\prime}}(0)=e_{f^{\prime}}(1)=2 n+1$. Thus, $\left|e_{f^{\prime}}(0)-e_{f^{\prime}}(1)\right| \leq 1$. Hence, $S\left(B_{n, n}\right)$ is SD-prime cordial.

Theorem 2.10. The graph $K_{1,3} * K_{1, n}$ is $S D$-prime cordial.

Proof. Let $V\left(K_{1,3} * K_{1, n}\right)=\{x, u, v, w\} \bigcup\left\{u_{i}, v_{i}, w_{i}: 1 \leq i \leq n\right\}$ and $E\left(K_{1,3} * K_{1, n}\right)=\{x u, x v, x w\} \bigcup\left\{u u_{i}, v v_{i}, w w_{i}: 1 \leq i \leq n\right\}$. Therefore, $K_{1,3} * K_{1, n}$ is of order $3 n+4$ and size $3 n+3$. Define $f: V\left(K_{1,3} * K_{1, n}\right) \rightarrow$ $\{1,2, \cdots, 3 n+4\}$ as follows:

$$
\begin{aligned}
& f(u)=1 ; \\
& f(v)=2 ; \\
& f(w)=4 \\
& f(x)=3 \\
& f\left(u_{i}\right)=3 i+2,1 \leq i \leq n \\
& f\left(v_{i}\right)=3 i+3,1 \leq i \leq n \\
& f\left(w_{i}\right)=3 i+4,1 \leq i \leq n .
\end{aligned}
$$

In view of the above labeling pattern we get,

$$
e_{f^{\prime}}(0)=\left\{\begin{array}{llll}
\frac{3 n+3}{2} & \text { if } & n & \text { is odd } \\
\frac{3 n+2}{2} & \text { if } & n & \text { is even } \\
\frac{3 n+3}{2} & \text { if } & n & \text { is odd } \\
\frac{3 n+4}{2} & \text { if } & n & \text { is even }
\end{array}\right.
$$

Thus, $\left|e_{f^{\prime}}(0)-e_{f^{\prime}}(1)\right| \leq 1$. Hence, $K_{1,3} * K_{1, n}$ is SD-prime cordial.

Theorem 2.11. The triangular ladder $T L_{n}$ is $S D$-prime cordial. 
Proof. Let $v_{1}, v_{2}, \cdots, v_{n}, u_{1}, u_{2}, \cdots, u_{n}$ be the vertices of $L_{n}$. Let $V\left(T L_{n}\right)=$ $\left\{u_{i}, v_{i}: 1 \leq i \leq n\right\}$ and $E\left(T L_{n}\right)=\left\{u_{i} u_{i+1}, v_{i} v_{i+1}, u_{i} v_{i+1}: 1 \leq i \leq\right.$ $n-1\} \bigcup\left\{u_{i} v_{i}: 1 \leq i \leq n\right\}$. Therefore, $T L_{n}$ is of order $2 n$ and size $4 n-3$. Define $f: V\left(T L_{n}\right) \rightarrow\{1,2, \cdots, 2 n\}$ as follows:

$$
\begin{gathered}
f\left(u_{i}\right)=2 i, 1 \leq i \leq n ; \\
f\left(v_{i}\right)=2 i-1,1 \leq i \leq n .
\end{gathered}
$$

In view of the above labeling pattern we get, $e_{f^{\prime}}(0)=2 n-2$ and $e_{f^{\prime}}(1)=2 n-1$.

Thus, $\left|e_{f^{\prime}}(0)-e_{f^{\prime}}(1)\right| \leq 1$. Hence, $T L_{n}$ is SD-prime cordial.

Theorem 2.12. The closed helm graph $\mathrm{CH}_{n}$ is $S D$-prime cordial.

Proof. Let $v$ be an apex vertex and $v_{1}, v_{2}, \cdots, v_{n}$ are rim vertices of $W_{n}$. Let $u_{1}, u_{2}, \cdots, u_{n}$ be the pendant vertices which are joined to each rim vertices of $W_{n}$ to obtained $H_{n}$. So $V\left(C H_{n}\right)=\{v\} \bigcup\left\{v_{i}, u_{i}: 1 \leq i \leq n\right\}$ and $E\left(C H_{n}\right)=\left\{v v_{i}, u_{i} v_{i}: 1 \leq i \leq n\right\} \bigcup\left\{v_{i} v_{i+1}, u_{i} u_{i+1}: 1 \leq i \leq n-\right.$ $1\} \bigcup\left\{v_{1} v_{n}, u_{1} u_{n}\right\}$. Therefore, $C H_{n}$ is of order $2 n+1$ and size $4 n$. Define $f: V\left(C H_{n}\right) \rightarrow\{1,2, \cdots, 2 n+1\}$ as follows:

$$
\begin{aligned}
& f(v)=1 \\
& f\left(v_{i}\right)=2 i, 1 \leq i \leq n \\
& f\left(u_{i}\right)=2 i+1, \quad 1 \leq i \leq n .
\end{aligned}
$$

In view of the above labeling pattern we get, $e_{f^{\prime}}(0)=e_{f^{\prime}}(1)=2 n$. Thus, $\left|e_{f^{\prime}}(0)-e_{f^{\prime}}(1)\right| \leq 1$. Hence, $C H_{n}$ is SD-prime cordial.

Theorem 2.13. The flower $F l_{n}$ is $S D$-prime cordial.

Proof. Let $V\left(F l_{n}\right)=\{v\} \bigcup\left\{v_{i}, u_{i}: 1 \leq i \leq n\right\}$ and $E\left(F l_{n}\right)=\left\{v v_{i}, v_{i} u_{i}, v u_{i}\right.$ : $1 \leq i \leq n\} \bigcup\left\{v_{n} v_{1}\right\} \bigcup\left\{v_{i} v_{i+1}: 1 \leq i \leq n-1\right\}$. Therefore, $F l_{n}$ is of order $2 n+1$ and size $4 n$. Define $f: V\left(F l_{n}\right) \rightarrow\{1,2, \cdots, 2 n+1\}$ as follows. Assign the labels to vertices $v, v_{i}, u_{i}$ as in theorem 2.14. We observe that, $e_{f^{\prime}}(0)=e_{f^{\prime}}(1)=2 n$.

Thus, $\left|e_{f^{\prime}}(0)-e_{f^{\prime}}(1)\right| \leq 1$. Hence, $F l_{n}$ is SD-prime cordial.

Theorem 2.14. The graph $P_{n}^{2}$ is $S D$-prime cordial. 
Proof. Let $v_{1}, v_{2}, \cdots, v_{n}$ be the vertices of the path $P_{n}$. Let $V\left(P_{n}^{2}\right)=$ $\left\{v_{1}, v_{2}, \cdots, v_{n}\right\}$ and $E\left(P_{n}^{2}\right)=\left\{v_{i} v_{i+1}: 1 \leq i \leq n-1\right\} \bigcup\left\{v_{i} v_{i+2}: 1 \leq\right.$ $i \leq n-2\}$. Therefore, $P_{n}^{2}$ is of order $n$ and size $2 n-3$. We define $f: V\left(P_{n}^{2}\right) \rightarrow\{1,2, \cdots, n\}$ by $f\left(v_{i}\right)=i$ for $1 \leq i \leq n$. We observe that, $e_{f^{\prime}}(0)=n-2$ and $e_{f^{\prime}}(1)=n-1$.

Thus, $\left|e_{f^{\prime}}(0)-e_{f^{\prime}}(1)\right| \leq 1$. Hence, $P_{n}^{2}$ is SD-prime cordial.

Remark 2.15. Note that $T\left(P_{n}\right)=P_{2 n-1}^{2}$. Therefore, $T\left(P_{n}\right)$ is also SDprime cordial.

Theorem 2.16. The graph obtained by duplication of each vertex by an edge in $P_{n}$ is $S D$-prime cordial.

Proof. Let $v_{1}, v_{2}, \cdots, v_{n}$ be the vertices of the path $P_{n}$ and $G$ be the graph obtained by duplication of each vertex $v_{i}$ of the path $P_{n}$ by an edge $v_{i}^{\prime} v_{i}^{\prime \prime}$ for $1 \leq i \leq n$ at a time. Let $V(G)=\left\{v_{i}, v_{i}^{\prime}, v_{i}^{\prime \prime}: 1 \leq i \leq n\right\}$ and $E(G)=\left\{v_{i} v_{i}^{\prime}, v_{i} v_{i}^{\prime \prime}, v_{i}^{\prime} v_{i}^{\prime \prime}: 1 \leq i \leq n\right\} \bigcup\left\{v_{i} v_{i+1}: 1 \leq i \leq n-1\right\}$. Therefore, $G$ is of order $3 n$ and size $4 n-1$. Define $f: V(G) \rightarrow\{1,2, \cdots, 3 n\}$ as follows:

$$
\begin{aligned}
& f\left(v_{2 i-1}\right)=6 i-4,1 \leq i \leq\left\lceil\frac{n}{2}\right\rceil ; \\
& f\left(v_{2 i}\right)=6 i-2,1 \leq i \leq\left\lfloor\frac{n}{2}\right\rfloor ; \\
& f\left(v_{2 i-1}^{\prime}\right)=6 i-5,1 \leq i \leq\left\lceil\frac{n}{2}\right\rceil ; \\
& f\left(v_{2 i}^{\prime}\right)=6 i-1,1 \leq i \leq\left\lfloor\frac{n}{2}\right\rfloor ; \\
& f\left(v_{i}^{\prime \prime}\right)=3 i, 1 \leq i \leq n .
\end{aligned}
$$

In view of the above labeling pattern we get, $e_{f^{\prime}}(1)=2 n$ and $e_{f^{\prime}}(0)=$ $2 n-1$.

Thus, $\left|e_{f^{\prime}}(0)-e_{f^{\prime}}(1)\right| \leq 1$. Hence, $G$ is SD-prime cordial.

Theorem 2.17. The graph $T\left(C_{n}\right)$ is $S D$-prime cordial.

Proof. Let $v_{1}, v_{2}, \cdots, v_{n}$ be the vertices of the cycle $C_{n}$. Let $V\left(T\left(C_{n}\right)\right)=$ $\left\{v_{i}, u_{i}: 1 \leq i \leq n\right\}$ and $E\left(T\left(C_{n}\right)\right)=\left\{v_{i} v_{i+1}, u_{i} u_{i+1}: 1 \leq i \leq n-1\right\} \bigcup\left\{v_{i} u_{i}\right.$ : $1 \leq i \leq n\} \bigcup\left\{v_{i} u_{i-1}: 2 \leq i \leq n\right\} \bigcup\left\{v_{n} v_{1}, u_{n} u_{1}, v_{1} u_{n}\right\}$. Therefore, $T\left(C_{n}\right)$ is of order $2 n$ and size $4 n$. Define $f: V\left(T\left(C_{n}\right)\right) \rightarrow\{1,2, \cdots, 2 n\}$ as follows:

$$
\begin{gathered}
f\left(v_{i}\right)=2 i-1 ; 1 \leq i \leq n \\
f\left(u_{i}\right)=2 i ; 1 \leq i \leq n
\end{gathered}
$$

In view of the above labeling pattern we get, $e_{f^{\prime}}(0)=e_{f^{\prime}}(1)=2 n$. Thus, $\left|e_{f^{\prime}}(0)-e_{f^{\prime}}(1)\right| \leq 1$. Hence, $T\left(C_{n}\right)$ is SD-prime cordial. 
Remark 2.18. Note that $T\left(C_{n}\right)=C_{2 n}^{2}$. It is natural to determine the $S D$-prime cordiality of $C_{2 n+1}^{2}, n \geq 2$.

Theorem 2.19. The graph obtained by duplication of each vertex by an edge in $C_{n}$ is $S D$-prime cordial.

Proof. Let $v_{1}, v_{2}, \cdots, v_{n}$ be the vertices of the cycle $C_{n}$ and $G$ be the graph obtained by duplication of each vertex $v_{i}$ of the cycle $C_{n}$ by an edge $v_{i}^{\prime} v_{i}^{\prime \prime}$ for $1 \leq i \leq n$. Then $V(G)=\left\{v_{i}, v_{i}^{\prime}, v_{i}^{\prime \prime}: 1 \leq i \leq n\right\}$ and $E(G)=E\left(C_{n}\right) \bigcup\left\{v_{i} v_{i}^{\prime}, v_{i} v_{i}^{\prime \prime}, v_{i}^{\prime} v_{i}^{\prime \prime}: 1 \leq i \leq n\right\}$. Therefore, $G$ is of order $3 n$ and size $4 n$. Define $f: V(G) \rightarrow\{1,2, \cdots, 3 n\}$ as follows:

$$
\begin{aligned}
& f\left(v_{2 i-1}\right)=6 i-5,1 \leq i \leq\left\lceil\frac{n}{2}\right\rceil ; \\
& f\left(v_{2 i}\right)=6 i-1,1 \leq i \leq\left\lfloor\frac{n}{2}\right\rfloor ; \\
& f\left(v_{2 i-1}^{\prime}\right)=6 i-4,1 \leq i \leq\left\lceil\frac{n}{2}\right\rceil ; \\
& f\left(v_{2 i}^{\prime}\right)=6 i-2,1 \leq i \leq\left\lfloor\frac{n}{2}\right\rfloor ; \\
& f\left(v_{i}^{\prime \prime}\right)=3 i, 1 \leq i \leq n .
\end{aligned}
$$

In view of the above labeling pattern we get, $e_{f^{\prime}}(0)=e_{f^{\prime}}(1)=2 n$. Thus, $\left|e_{f^{\prime}}(0)-e_{f^{\prime}}(1)\right| \leq 1$. Hence, $G$ is SD-prime cordial.

Theorem 2.20. The quadrilateral snake $Q_{n}$ is $S D$-prime cordial.

Proof. Let $v_{1}, v_{2}, \cdots, v_{n}$ be the vertices of path $P_{n}$. Let $V\left(Q_{n}\right)=$ $V\left(P_{n}\right) \bigcup\left\{u_{i}, w_{i}: 1 \leq i \leq n-1\right\}$ and $E\left(Q_{n}\right)=E\left(P_{n}\right) \bigcup\left\{v_{i} u_{i}, u_{i} w_{i}, v_{i+1} w_{i}\right.$ : $1 \leq i \leq n-1\}$. Therefore, $Q_{n}$ is of order $3 n-2$ and size $4 n-4$. Define $f: V\left(Q_{n}\right) \rightarrow\{1,2, \cdots, 3 n-2\}$ as follows:

$$
\begin{aligned}
& f\left(v_{i}\right)= \begin{cases}3 i-2 & \text { if } i \text { is odd and } 1 \leq i \leq n \\
3 i-3 & \text { if } i \text { is even and } 1 \leq i \leq n\end{cases} \\
& \mathrm{f}\left(\mathrm{u}_{i}\right)=3 i-1,1 \leq i \leq n-1 ;
\end{aligned}
$$

In view of the above labeling pattern we get, $e_{f^{\prime}}(0)=e_{f^{\prime}}(1)=2 n-2$. Thus, $\left|e_{f^{\prime}}(0)-e_{f^{\prime}}(1)\right| \leq 1$. Hence, $Q_{n}$ is SD-prime cordial.

Theorem 2.21. An alternate triangular snake $A\left(T_{n}\right)$ is $S D$-prime cordial. 
Proof. Let $v_{1}, v_{2}, \cdots, v_{n}$ be the vertices of path $P_{n}$. The graph $A\left(T_{n}\right)$ is obtained by joining the vertices $v_{i} v_{i+1}$ (alternately) to a new vertex $u_{i}, 1 \leq i \leq n-1$ for even $n$ and $1 \leq i \leq n-2$ for odd $n$. Therefore, $\mathrm{V}\left(\mathrm{A}\left(\mathrm{T}_{n}\right)\right)=V\left(P_{n}\right) \bigcup\left\{u_{i}: 1 \leq i \leq\left\lfloor\frac{n}{2}\right\rfloor\right\}$ and $E\left(A\left(T_{n}\right)\right)=E\left(P_{n}\right) \bigcup\left\{v_{2 i-1} u_{i}:\right.$ $\left.1 \leq i \leq\left\lfloor\frac{n}{2}\right\rfloor ; u_{i} v_{2 i}: 1 \leq i \leq\left\lfloor\frac{n}{2}\right\rfloor\right\}$. Also,

$$
\begin{aligned}
& \left|V\left(A\left(T_{n}\right)\right)\right|= \begin{cases}\frac{3 n-1}{2} & \text { if } n \text { is odd } \\
\frac{3 n}{2} & \text { if } n \text { is even } \\
2 n-2 & \text { if } n \text { is odd } \\
2 n-1 & \text { if } n \text { is even }\end{cases}
\end{aligned}
$$

Case 1: $n$ is odd.

Define $f: V\left(A\left(T_{n}\right)\right) \rightarrow\left\{1,2, \cdots, \frac{3 n-1}{2}\right\}$ as follows:

$$
\begin{aligned}
& f\left(v_{i}\right)= \begin{cases}3 i-2 & \text { if } i \text { is odd } \\
3 i-1 & \text { if } i \text { is even } ;\end{cases} \\
& f\left(u_{i}\right)=3 i, \quad 1 \leq i \leq\left\lfloor\frac{n}{2}\right\rfloor .
\end{aligned}
$$

We observe that, $e_{f^{\prime}}(0)=e_{f^{\prime}}(1)=n-1$.

Case 2: $n$ is even.

Define $f: V\left(A\left(T_{n}\right)\right) \rightarrow\left\{1,2, \cdots, \frac{3 n}{2}\right\}$ as follows:

$$
\begin{aligned}
& f\left(v_{i}\right)= \begin{cases}3 i-1 & \text { if } i \text { is odd } \\
3 i & \text { if } i \text { is even } ;\end{cases} \\
& f\left(u_{i}\right)=3 i-2,1 \leq i \leq\left\lfloor\frac{n}{2}\right\rfloor .
\end{aligned}
$$

We observe that, $e_{f^{\prime}}(0)=n-1$ and $e_{f^{\prime}}(1)=n$.

Thus, in both cases $\left|e_{f^{\prime}}(0)-e_{f^{\prime}}(1)\right| \leq 1$. Hence, $A\left(T_{n}\right)$ is SD-prime cordial.

Theorem 2.22. The comb $P_{n} \odot K_{1}$ is SD-prime cordial.

Proof. Let $v_{1}, v_{2}, \cdots, v_{n}$ be the vertices of path $P_{n}$. Let $V\left(P_{n} \odot K_{1}\right)=$ $\left\{v_{i}, u_{i}: 1 \leq i \leq n\right\}$ and $E\left(P_{n} \odot K_{1}\right)=\left\{v_{i} v_{i+1}: 1 \leq i \leq n-1\right\} \bigcup\left\{v_{i} u_{i}:\right.$ $1 \leq i \leq n\}$. Therefore, $P_{n} \odot K_{1}$ is of order $2 n$ and size $2 n-1$. Define 
$f: V\left(P_{n} \odot K_{1}\right) \rightarrow\{1,2, \cdots, 2 n\}$ as follows:

$$
\begin{aligned}
& f\left(v_{i}\right)=2 i-1,1 \leq i \leq n \\
& f\left(u_{i}\right)=2 i, 1 \leq i \leq n .
\end{aligned}
$$
$n$.

In view of the above labeling pattern we get, $e_{f^{\prime}}(0)=n-1$ and $e_{f^{\prime}}(1)=$ Thus, $\left|e_{f^{\prime}}(0)-e_{f^{\prime}}(1)\right| \leq 1$. Hence $P_{n} \odot K_{1}$ is SD-prime cordial.

Corollary 2.23. The crown $C_{n} \odot K_{1}$ is $S D$-prime cordial.

Theorem 2.24. The jewel $J_{n}$ is $S D$-prime cordial.

Proof. Let $V\left(J_{n}\right)=\{u, v, x, y\} \bigcup\left\{u_{i}: 1 \leq i \leq n\right\}$ and $E\left(J_{n}\right)=$ $\{u x, u y, x y, v x, v y\} \bigcup\left\{u u_{i}, v u_{i}: 1 \leq i \leq n\right\}$. Therefore, $J_{n}$ is of order $n+4$ and size $2 n+5$. Define $f: V\left(J_{n}\right) \rightarrow\{1,2, \cdots, n+4\}$ as follows:

$$
\begin{aligned}
& f(u)=1 ; \\
& f(v)=2 \\
& f(x)=3 \\
& f(y)=4 \\
& f\left(u_{i}\right)=i+4, \quad 1 \leq i \leq n .
\end{aligned}
$$

In view of the above labeling pattern we get, $e_{f^{\prime}}(0)=n+2$ and $e_{f^{\prime}}(1)=$ $n+3$.

Thus, $\left|e_{f^{\prime}}(0)-e_{f^{\prime}}(1)\right| \leq 1$. Hence $J_{n}$ is SD-prime cordial.

\section{References}

[1] J. A. Gallian, A Dyamic Survey of Graph Labeling, The Electronic J. Combin., 17, (2014) \# DS6.

[2] F. Harary, Graph Theory, Addison-wesley, Reading, Mass, (1972).

[3] G. C. Lau, H. H. Chu, N. Suhadak, F. Y. Foo and H. K. Ng, On SD-Prime Cordial Graphs, International Journal of Pure and Applied Mathematics, 106 (4), pp. 1017-1028, (2016).

[4] G. C. Lau and W. C. Shiu, On SD-Prime Labeling of Graphs, Utilitas Math., accepted. 
[5] G. C. Lau, W.C. Shiu, H.K. Ng, C. D. Ng and P. Jeyanthi, Further Results on SD-Prime Labeling, JCMCC, 98, pp. 151-170, (2016).

\author{
A. Lourdusamy \\ Department of Mathematics, \\ St. Xavier's College (Autonomous), \\ Palayamkottai-627002, \\ Tamilnadu, \\ India \\ e-mail : lourdusamy15@gmail.com \\ and \\ F. Patrick \\ Department of Mathematics, \\ St. Xavier's College (Autonomous), \\ Palayamkottai-627002, \\ Tamilnadu, \\ India \\ e-mail : patrick881990@gmail.com
}

